\title{
Trees associated with the configuration of Herman rings
}

\author{
MITSHUHIRO SHISHIKURA \\ Department of Mathematics, Kyoto University, Kyoto, 606, Japan†
}

(Received 8 August 1987 and revised 10 October 1987)

\begin{abstract}
For a rational function with Herman rings, we define a tree and a piece-wise linear map on it, which reflect the configuration of the Herman rings. Their properties are investigated and some examples are given. Moreover, it is possible to define a similar tree associated with (super)attractive basins or Siegel disks.
\end{abstract}

\section{Introduction}

We are concerned with the dynamics of rational functions on the Riemann sphere $\overline{\mathbb{C}}=\mathbb{C} \cup\{\infty\}$ (see [B], for a review). Douady and Hubbard [DH] described the dynamics of polynomials with connected and locally connected Julia sets, in terms of so-called Hubbard's trees. However, if the Julia set $J_{f}$ of a rational function $f$ is disconnected, then the complement $\overline{\mathbb{C}}-J_{f}$ divides $J_{f}$ into an infinite number of connected components in a complicated manner. The aim of this paper is to propose a new kind of trees which reflect this situation, for rational functions with Herman rings. This construction of the tree can be generalized to rational functions with (super)attractive basins or Siegel disks.

A Herman ring of period $p$ of a rational function $f$ is a connected component $A$ of $\overline{\mathbb{C}}-J_{f}$ such that $f^{p}(A)=A, f^{j}(A) \cap A=\varnothing(0<j<p)$ and $f^{p}$ on $A$ is conformally conjugate to an irrational rotation $z \rightarrow e^{2 \pi i \theta} z$ on a concentric annulus $\{z \in \mathbb{C}|r<| z \mid<1\}$ with $0<r<1$ and $\theta \in \mathbb{R}-\mathbb{Q}$. (see [D, H].) Let $\gamma$ be an oriented simple closed curve in $A$ corresponding to $\left\{z \in \mathbb{C}|| z \mid=r^{\prime}\right\}\left(r<r^{\prime}<1\right)$. Then $\gamma_{j}=f^{j-1}(\gamma)(j=1, \ldots, p)$ are invariant curves in the sense that $f\left(\gamma_{j}\right)=\gamma_{j+1}(j=1, \ldots, p-1), f\left(\gamma_{p}\right)=\gamma_{1}$ and that $f$ respects their orientations.

If $p \geq 2$, there naturally arises the problem of the configuration of $\gamma_{j}$ or rather of the Herman rings. Here, a configurations means a cyclically ordered collection of disjoint, oriented simple closed curves on $\bar{C}$, up to orientation preserving homeomorphism of $\overline{\mathbb{C}}$ and simultaneous change of orientations of all curves. Let us see simple examples.

In the case of $p=2$, there are two possibilities which are indicated in figure 1.

In the case of $p=3$, there still are two possibilities, even if one does not take into account the cyclic order and the orientation of $\gamma_{i}$ (see figure 2). 

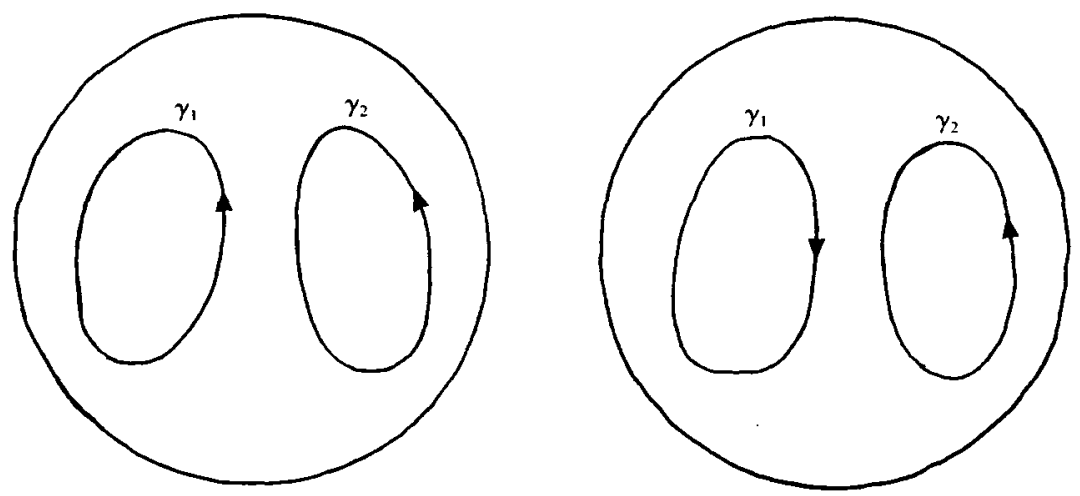

FIGURE 1
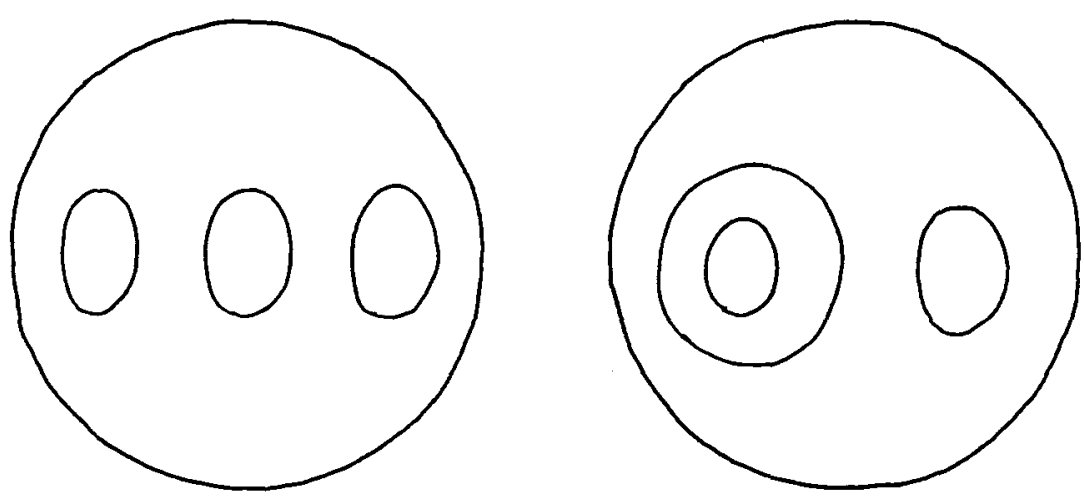

Figure 2

The tree $T_{f}$, which will be defined in $\S 3$, describes the configuration of Herman rings and their pre-images, and also involves some quantitative information, such as the moduli of the rings. An induced map $f_{*}$ on $T_{f}$ roughly describes the dynamics of $f$. Furthermore, it turns out that $f_{*}$ has some remarkable features: $f_{*}$ is piece-wise linear (with respect to a certain metric) and its derivatives are positive integers. Hence, it is considerably easier to deal with $\left(T_{f}, f_{*}\right)$ than $(\overline{\mathbb{C}}, f)$ itself.

Moreover, the converse process is possible in many cases, i.e. one can construct a rational function, by means of surgery developed in [S1], from a given tree with these properties and satisfying certain conditions. We call this the realization problem, where we say a rational function $f$ realizes a pair $(T, F)$ of a tree and a map, if $\left(T_{f}, f_{*}\right)$ is conjugate to $(T, F)$ by an isometry. The realization problem will be discussed in another paper [S2]. In addition, there is a formula which gives a lower bound on the degree of rational functions realizing $(T, F)$.

We proceed as follows. In $\S 1$, we review well-known facts about annuli (Lemma 1.3 ) and prepare some notations. In $\S 2$, we propose an abstract construction of a tree from a collection of annuli satisfying the Condition $(*)$, although the proofs of the statements are postponed until $\S 7$. We define, in $\S 3$, the tree $T_{f}$ and the map $f_{*}$ associated with the configuration of Herman rings of $f$. Their basic properties 
are examined also in $\S 3$. Stated in $\S 4$ are some consequences of the basic properties. In $\S 5$, examples of the trees satisfying the basic properties are given. We discuss in $\$ 6$ how we can generalize the construction of the tree, for example, to rational functions with (super)attractive basins or Siegel disks.

\section{Annulus}

In the following, we shall say simply 'components' instead of 'connected components'.

Definition 1.1. A connected open set $A \subset \overline{\mathbb{C}}$ is called an annulus, if $A^{\mathrm{c}}=\overline{\mathbb{C}}-A$ has exactly two components, neither of which is a point.

Definition 1.2. Let $X$ and $Y$ be subsets (or points) of $\overline{\mathbb{C}}$ and $A$ an annulus (resp. $\gamma$ a simple closed curve in $\bar{C}$ ). We say that $A$ (resp. $\gamma$ ) separates $X$ and $Y$, if one component of $A^{c}$ (resp. $\gamma^{c}$ ) contains $X$ and the other $Y$.

We also say that $A$ (resp. $\gamma$ ) separates a single set $X$, if $X$ intersects both components of $A^{c}$ (resp. $\gamma^{c}$ ).

LeMma 1.3. Let $A$ be an annulus.

(i) There exists an $r=r_{A}$ with $0<r<1$ and a conformal mapping $\phi=\phi_{A}$ :A $\rightarrow$ $\{z \in \mathbb{C}|r<| z \mid<1\}$.

(ii) If $\phi_{1}: A \rightarrow\left\{r^{\prime}<|z|<1\right\}$ is another conformal mapping, then $r=r^{\prime}$ and $\phi_{1}=c^{\phi}$ or $c r / \phi$, where $c$ is a constant with $|c|=1$. Hence the $r_{A}$ is uniquely determined.

See [A1] for the proof. From now on, $\phi_{A}$ and $r_{A}$ always denote those as above.

Definition 1.4. The modulus of an annulus $A$ is

$$
m(A)=-\frac{1}{2 \pi} \log r_{A}
$$

As a convention, we define $m(\phi)=0$, for the empty $\phi$.

Definition 1.5. Let $A$ be an annulus. Define

$$
\begin{aligned}
A[z] & =\phi_{A}^{-1}\left(\left\{\zeta|| \zeta|=| \phi_{A}(z) \mid\right\}\right) \quad \text { for } z \in A, \\
A(x, y) & =\{z \in A \mid A[z] \quad \text { separates } x \text { and } y\} .
\end{aligned}
$$

The following two lemmas can be readily proved using Lemma 1.3.

LeMma 1.6. (i) In the above definition, $A[z]$ hence $A(x, y)$ do not depend on the choice of $\phi_{A}$. For $z \in A, A[z]$ is a simple closed curve in $A$ containing $z$ and separating วA. $A(x, y)$ is either empty or an annulus separating $x$ and $y$.

(ii) $m(A(x, y))=$ the length of

$$
\left\{-\frac{1}{2 \pi} \log \left|\phi_{A}(z)\right| \mid z \in A(x, y)\right\}(\subset \mathbb{R}) .
$$

LeMMA 1.7. Let $f: A_{1} \rightarrow A_{2}$ be an analytic covering map of annuli of degree $d$. Then $d \cdot m\left(A_{1}\right)=m\left(A_{2}\right)$ and $\phi_{A_{2}}(f(z))=\left(\phi_{A_{1}}(z)\right\}^{d}$, for suitable choice of $\phi_{A_{i}}$. Hence $f\left(A_{1}\{z\}\right)=A_{2}[f(z)]$ for $z \in A_{1}$. 
Lemma 1.8. (The Composition Law.) Let $A_{i}(i=1,2, \ldots)$ be disjoint annuli contained in an annulus $A$. Suppose each $A_{i}$ separates $A^{c}$. Then

$$
\sum_{i} m\left(A_{i}\right) \leq m(A) \text {. }
$$

See [A2] for the proof. This inequality follows from the principle of extremal length. In fact, one can prove it using the equality

$$
m(A)=\inf _{\rho} \iint_{A} \rho^{2} d x d y,
$$

where the infimum is over Borel measurable functions $\rho(z) \geq 0$ satisfying $\int_{\gamma} \rho|d z| \geq 1$ for any locally rectifiable closed curve $\gamma$ separating $\partial A$.

\section{Abstract tree}

All the statements in this section will be proved in $\S 7$.

Let $\mathscr{A}$ be a collection of disjoint annuli in $\bar{C}$. Throughout this section (except (2.10)), we assume the following Condition (*).

Condition (*). There exist a non-empty closed set $B \subset \bar{C}$ such that:

$B$ consists of a finite number of connected components $B_{1}, \ldots, B_{N}$, none of which is a point;

Each $A \in \mathscr{A}$ is disjoint with $B$ and separates $B$. (i.e., $A$ separates $B_{i}$ and $B_{j}$ for some $i, j$.) Let

$$
\begin{gathered}
\Gamma=\Gamma(\mathscr{A})=\{A[z] \mid A \in \mathscr{A}, z \in A\}, \\
\bigcup \mathscr{A}=\bigcup_{A \in \mathscr{A}} A .
\end{gathered}
$$

In the following, $\boldsymbol{A}, \boldsymbol{A}_{i}$ (resp. $\gamma, \gamma_{i}, \ldots$ ) always denote elements of $\mathscr{A}$ (resp. $\Gamma$ ). We are going to define a tree $T(\mathscr{A})$.

Definition 2.1. For $x, y \in \overline{\mathbb{C}}$,

$$
d(x, y)=\sum_{A \in \mathscr{A}} m(A(x, y)) .
$$

LeMMA 2.2. $\sum_{A \in \mathscr{A}} m(A)<\infty$. Hence $d(x, y)<\infty$, for any $x, y$.

LEMMA 2.3.

$$
\begin{aligned}
& d(x, x)=0, \quad d(x, y)=d(y, x), \\
& d(x, z) \leq d(x, y)+d(y, z) .
\end{aligned}
$$

LEMMA 2.4. $d(\cdot, \cdot)$ is continuous on $\overline{\mathbb{C}} \times \overline{\mathbb{C}}$.

Definition 2.5. Let us define $\sim_{\mathscr{A}}, T(\mathscr{A}), \pi$ and $\bar{d}$ as follows:

$$
\begin{gathered}
x \sim \mathscr{A} \text { for } x, y \in \overline{\mathbb{C}} \text { if and only if } d(x, y)=0 ; \\
T(\mathscr{A})=\overline{\mathbb{C}} / \sim
\end{gathered}
$$

$\pi: \overline{\mathbb{C}} \rightarrow T(\mathscr{A})$ is the natural projection. For $x, y \in T(\mathscr{A}), \bar{d}(x, y)=d(\tilde{x}, \tilde{y})$, where $\tilde{x} \in \pi^{-1}(x), \tilde{y} \in \pi^{-1}(y)$. We call $T(\mathscr{A})$ the tree associated with the configuration $\mathscr{A}$. 
Concerning these definitions, the following lemma can be proved without difficulty using Lemmas 2.3 and 2.4.

LEMMA 2.6. The relation $\sim \mathscr{A}$ is an equivalence relation, hence $T(\mathscr{A})$ is well-defined. The $\bar{d}(\cdot, \cdot)$ is also well-defined and is a metric on $T(\mathscr{A})$. Furthermore, the quotient topology on $T(\mathscr{A})$ is equivalent to the topology defined by $\bar{d}$.

Definition 2.7. A tree is a one-dimensional (finite) simplicial complex which is connected and contains no loop (i.e. is simply connected). Let $T$ be a tree. For $x \in T$, a component of $T-\{x\}$ is called a branch at $x$, and $\mathscr{B}_{x}$ denotes the set of branches at $x$. A point $x$ is an end point if $\# \mathscr{B}_{x}=1$, branch point if $\# \mathscr{B}_{x} \geq 3$. An arc is a subset homeomorphic to a closed interval. Its interior and end points, corresponding to those of the interval, are denoted by int $L, \partial L$ for the arc $L$. A metric on $T$ is called linear, if every arc in $T$ is isometric to an interval with the standard metric. This is equivalent to the following: if $y$ is on an arc joining $x$ and $z$, then $d(x, z)=$ $d(x, y)+d(y, z)$.

As for the $T(\mathscr{A})$ defined in Definition 2.5, we have:

THEOREM 2.8. (a) If $\mathscr{A}$ is a collection of disjoint annuli satisfying the Condition (*), then

( $\alpha) T(\mathscr{A})$ is a tree.

$(\beta) \bar{d}(\cdot, \cdot)$ on $T(\mathscr{A})$ is a linear metric.

$(\gamma)$ The projections $\pi(A)(A \in \mathscr{A})$ are dense in $T(\mathscr{A})$.

( $\delta)$ Each end point of $T(\mathscr{A})$ is $\pi\left(B_{i}\right)$ for some $i$.

Although the proof is given in $\S 7$, figure 3 might convince you of the assertion $(\alpha)$. For the argument in the next section, we need the following lemma.

LemMA 2.9. If $x \in A$ and $y \notin A[x]$, then $d(x, y)>0$. Hence $\pi^{-1}(\pi(x))=A[x]$ and $\pi^{-1}(\pi(A))=A$. Moreover $\pi(A)$ is isometric to an open interval.

Degenerate case 2.10 . In the Condition (*), we can eliminate the assumption that none of the components of $B$ is a point. Then a similar construction yields a tree $T(\mathscr{A})$ which may be of infinite length. In this case, we have to change the above statements as follows:

LEMMA $2.2^{\prime}$. There exists a finite set $B_{\infty}$ consisting of one point components of $B$ such that

$$
\begin{array}{ll}
d(x, y)<\infty & \text { for } \quad x, y \in \overline{\mathbb{C}}-B_{\infty}, \\
d(x, y)=\infty & \text { for } \quad x \in \overline{\mathbb{C}}, y \in B_{\infty} \quad \text { with } x \neq y .
\end{array}
$$

LEMMA $2.4^{\prime} . \bar{d}$ is a metric on $T(\mathscr{A})-\pi\left(B_{\infty}\right)=\pi\left(\overline{\mathbb{C}}-B_{\infty}\right)$. The quotient topology on $\pi\left(\overline{\mathbb{C}}-B_{\infty}\right)$ is equivalent to the topology defined by $\bar{d}$.

As the topology of $T(\mathscr{A})$, we adopt the quotient topology, since $\bar{d}$ cannot be a metric on the whole $T(\mathscr{A})$. The points of $\pi\left(B_{\infty}\right)$ are called points at infinity. 

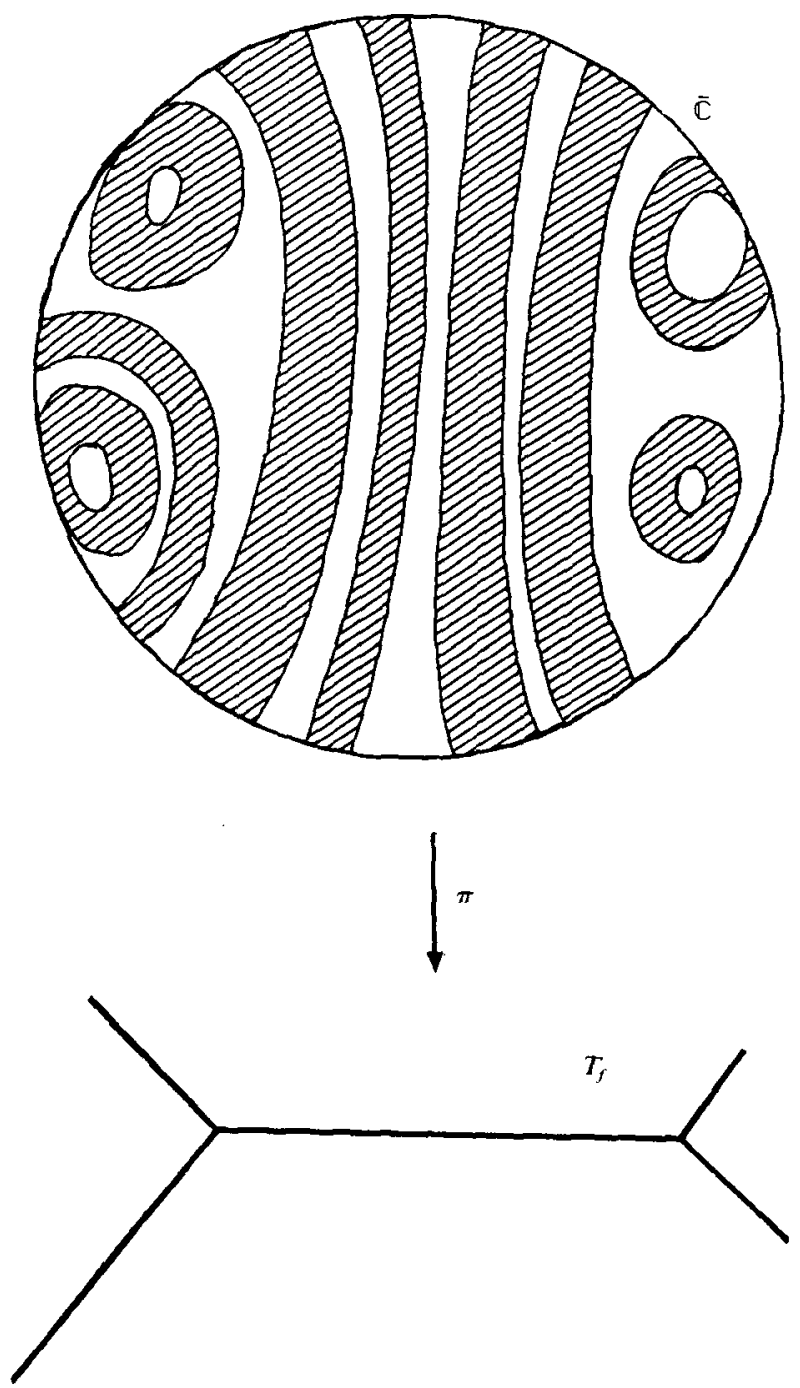

Figure 3

THEOREM $2.8\left(\mathrm{~b}^{\prime}\right) . \bar{d}$ on $\pi\left(\overline{\mathbb{C}}-B_{\infty}\right)$ is $、$ linear metric.

3. Tree associated with Herman rings

Let $f$ be a rational function which has Herman rings.

Definition 3.1. Let

$\mathscr{A}_{0}=\{$ components of (Herman rings - the closure of the forward orbits of critical points)\};

$$
\begin{aligned}
\mathscr{A}^{\prime} & =\left\{\text { components of } f^{-n}(A) \mid A \in \mathscr{A}_{0}, n \geq 0\right\} ; \\
B & =\text { the union of the boundaries of Herman rings; } \\
\mathscr{A} & =\left\{A \in \mathscr{A}^{\prime} \mid f^{n}(A) \text { separates } B \text { for all } n \geq 0\right\} .
\end{aligned}
$$


Lemma 3.2. $\mathscr{A}_{0}, \mathscr{A}^{\prime}$ and $\mathscr{A}$ are collections of annuli. If $A \in \mathscr{A}$, then $f(A) \in \mathscr{A}$ and $\left.f\right|_{A}: A \rightarrow f(A)$ is a covering map. If $\gamma \in \Gamma=\Gamma(\mathscr{A})$, then $f(\gamma) \in \Gamma$. Furthermore, each component of $f^{-1}(\gamma)$ either belongs to $\Gamma$ or do not intersect with any element of $\Gamma$, according to whether it separates $B$ or not.

Proof. Because of the dynamics on the Herman rings, $\mathscr{A}_{0}$ consists of annuli. It is clear that if $A$ is an annulus containing no orbit of critical point, then $f^{n}: f^{-n}(A) \rightarrow A$ is a covering map. Therefore $f^{-n}(A)$ is a disjoint union of annuli. So $\mathscr{A}^{\prime}$ consists of annuli and the definition of $\mathscr{A}$ does make sense. The proof of the rest is easy (cf. Lemma 1.7).

Lemma And Definition 3.3. The $\mathscr{A}$ satisfies Condition (*) with the above B. Hence, according to $\S 2$, we can define the tree

$$
T_{f}=T(\mathscr{A}) \text {. }
$$

This $T_{f}$ is called the tree associated with Herman rings of $f$.

Proof. Note that a boundary component of a Herman ring cannot be a point, in view of the removable singularity theorem. So it is easy to verify the Condition $(*)$.

Definition 3.4. Define $f_{*}: T_{f} \rightarrow T_{f}$ by

$$
f_{*}(x)=\pi \circ f\left(\partial \pi^{-1}(x)\right),
$$

where $\partial \pi^{-1}(x)$ is the boundary of $\pi^{-1}(x)$ in $\overline{\mathbb{C}}$.

LEMMA 3.5. $f_{*}$ is well-defined and continuous.

Proof. We show that

$$
\text { any } \gamma \in \Gamma \text { does not separate } j\left(\partial \pi^{-1}(x)\right) \text { for } x \in T_{f} \text {. }
$$

Then it follows that $\pi \circ f\left(\partial \pi^{-1}(x)\right)$ is a point and $f_{*}$ is well-defined.

Let $\gamma_{i}(i=1, \ldots, m)$ and $\gamma_{j}^{\prime}(j=1, \ldots, n)$ be components of $f^{-1}(\gamma)$, which are simple closed curves, such that $\gamma_{i}$ separate $B$ and $\gamma_{j}^{\prime}$ do not. It follows from Lemma 3.2 that $\gamma_{i} \in \Gamma$ and $\gamma_{j}^{\prime} \notin \Gamma$. So $\gamma_{i}$ cannot separate $\pi^{-1}(x)$, hence $\partial \pi^{-1}(x)$ neither. On the other hand, for each $\gamma_{j}^{\prime}$, there exists a component $C_{j}$ of $\left(\gamma_{j}^{\prime}\right)^{c}$ containing $B$. Every $\gamma^{\prime} \in \Gamma$ is contained in $C_{j}$, since it must separate $B$ and does not intersect $\gamma_{j}^{\prime}$ by Lemma 3.2. So $d\left(z, z^{\prime}\right)=0$, for any $z, z^{\prime} \in C_{j}^{c}$. Then either $\pi^{-1}(x) \cap C_{j}^{c}=\phi$ or $C_{j}^{c} \subset \pi^{-1}(x)$. In any case, $\partial \pi^{-1}(x) \subset \bar{C}_{j}$.

Thus neither $\gamma_{i}$ nor $\gamma_{j}^{\prime}$ separates $\partial \pi^{-1}(x)$. There exists a component $C$ of $f^{-1}\left(\gamma^{c}\right)=$ $\left(\bigcup_{i} \gamma_{i} \cup \bigcup_{j} \gamma_{j}^{\prime}\right)^{c}$, whose closure contains $\partial \pi^{-1}(x)$. Since $f(C)$ is a component of $\gamma^{c}, \gamma$ cannot separate $f\left(\partial \pi^{-1}(x)\right) \subset f(\vec{C})$. The assertion is proved.

Note that if $f_{*}(x) \neq \pi(\gamma)$, then $\pi(C)$ is a neighborhood of $x$ and as shown above, $f_{*}(\pi(C))$ is a component of $T_{f}-\pi(\gamma)$. It follows that $f_{*}$ is continuous, since finite intersections of components of $T_{f}-\pi(\gamma)(\gamma \in \Gamma)$ form a basis of open sets by Theorem $2.8(\gamma)$.

THEOREM 3.6. Write $T=T_{f}, F=f_{*}$ and $d=\bar{d}$. Then $(T, d, F)$ satisfies the following.

(a) $(T, d)$ is a tree with a linear metric.

(b) $F: T \rightarrow T$ is continuous. 
(c) There exist a finite subset $\operatorname{Sing}(T, F)$ of $T$ and a function $D F: T$-Sing $(T, F) \rightarrow$ $\mathbb{N}(=$ the set of positive integers $)$, which is constant on each component, such that: if $x$ and $y$ are in the same component $T^{\prime}$ of $T$-sing $(T, F)$, then $d(F(x), F(y))=$ $k \cdot d(x, y)$, where $k=\left.D F\right|_{T}$.

(d) There exist subarcs $I_{i j}\left(i=1, \ldots, l ; j \in Z / p_{i} Z\right)$ of $T$ such that:

int $I_{i j}$ are disjoint and do not contain branch points;

$$
F\left(I_{i j}\right)=I_{i j+1} ; \text { and } F^{p_{i}}=i d \text { on } I_{i j} \text {. }
$$

(e) $T=\bigcup_{n \geq 0} \bigcup_{i, j} F^{-n}$ (int $I_{i j}$ ).

(f) Each end point of $T$ is an end point of an $I_{i j}$.

In fact we may take

Sing $\left(T_{f}, f_{*}\right)=\left\{\right.$ end points, branch points of $\left.T_{f}\right\} \cup \pi$ critical points of $\left.f\right\}$;

$D f_{*}$ on $T^{\prime}=$ the degree of the covering $f: \pi^{-1}(x) \rightarrow \pi^{-1}\left(f_{*}(x)\right)$, where $x$ is any point of $T^{\prime} \cap \pi(\bigcup \mathscr{A})$ (this is later proved to be well-defined);

$$
\left\{I_{i j}\right\}=\{\pi(\bar{A}) \mid A \text { is a Herman ring of } f\},
$$

where $I_{i j}$ inherit the cyclic order of the Herman rings, and $l$ is the number of cycles of Herman rings and $p_{i}$ the periods.

Proof. The assertions (a), (e) and (f) immediately follow from Theorem 2.8, and (b) from Lemma 3.5. Also (d) follows from the property of Herman rings, provided that the subscripts are suitably chosen. Before proving (c), we state the following lemma.

LEMMA 3.7. Let $R$ be an annulus bounded by real analytic simple closed curves $\gamma_{1}$, $\gamma_{2}$ and $g$ an analytic map from a neighborhood of $\bar{R}$ to $\bar{C}$. Suppose that $g$ has no critical point in $\bar{R}, \gamma_{i}^{\prime}=g\left(\gamma_{i}\right)$ are real analytic simple closed curves and $\left.g\right|_{\gamma_{i}}: \gamma_{i} \rightarrow \gamma_{i}^{\prime}$ is a covering of degree $k_{i}(i=1,2)$.

Then $k_{1}=k_{2}, g(R)$ is an annulus bounded by $\gamma_{i}^{\prime}$, and $g: R \rightarrow g(R)$ is a covering of degree $k_{1}$.

Proof. Extend $g$ to a branched cover (or 'quasi-regular map') $g_{1}: \overline{\mathbb{C}} \rightarrow \overline{\mathbb{C}}$, by glueing along each $\gamma_{i}$ the map $z \rightarrow z^{k_{i}}$ on $\{|z|<1\}$, so that in each component $R^{c}, g_{1}$ has a unique critical point of multiplicity $k_{i}-1$ (or no critical point if $k_{i}=1$ ). Let $d$ be the degree of $g_{1}$. Considering the degree and the number of critical points, we have

$$
d \geq k_{i} \text { and } 2(d-1) \leqq\left(k_{1}-1\right)+\left(k_{2}-1\right) .
$$

Hence $k_{1}=k_{2}=d$.

Moreover there are homeomorphisms $h, h^{\prime}$ of $\overline{\mathbb{C}}$ such that

$$
h \circ g_{1} \circ h^{\prime}(z)=z^{d} \text {. }
$$

Then it is easy to see that $\gamma_{1}^{\prime} \cap \gamma_{2}^{\prime}=\phi$, and $g(R)$ is the annulus bounded by $\gamma_{i}^{\prime}$, etc.

Proof of Theorem 3.6(c). Fix a component $T^{\prime}$ of $T_{f}$-Sing $\left(T_{f}, f_{*}\right)$, which is an open arc. Let $x, y$ be any two points of $T^{\prime} \cap \pi(\bigcup \mathscr{A})$ and $J$ the arc joining $x$ and $y$. Then $J \subset T^{\prime}$ and $R=\pi^{-1}$ (int $J$ ) is an annulus bounded by $\gamma_{1}=\pi^{-1}(x)$ and $\gamma_{2}=\pi^{-1}(y)$. By the definition of Sing $\left(T_{f}, f_{*}\right), f$ does not have a critical point in $\bar{R}$, and the 
assumption of Lemma 3.7 is fulfilled. Hence, the degree of the covering $f: \pi^{-1}(x) \rightarrow$ $\pi^{-1}\left(f_{*}(x)\right)$ is the same as that for $y$. This implies that $D F$ is well-defined and constant on $T^{\prime}$. Let $k=\left.D F\right|_{T^{\prime}}$.

If $\gamma \in \Gamma$ separates $\gamma_{1}$ and $\gamma_{2}$, then $\gamma \subset R$ and $f(\gamma)$ separates $f\left(\gamma_{1}\right)=\pi^{-1}\left(f_{*}(x)\right)$ and $f\left(\gamma_{2}\right)=\pi^{-1}\left(f_{*}(y)\right)$, by Lemma 3.7. Conversely if $\gamma \in \Gamma$ separates $f\left(\gamma_{1}\right)$ and $f\left(\gamma_{2}\right)$, then $y \subset f(R)$ and there exists a unique component of $f^{-1}(\gamma)$ which separates $\gamma_{1}$ and $\gamma_{2}$. Thus $f$ gives a one to one correspondence between $\left\{\gamma \in \Gamma\right.$ separating $\gamma_{1}$ and $\left.\gamma_{2}\right\}$ and $\left\{\gamma^{\prime} \in \Gamma\right.$ separating $f\left(\gamma_{1}\right)$ and $\left.f\left(\gamma_{2}\right)\right\}$. (But note here that $f(R)$ may contain a $\gamma \in \Gamma$ which does not separate $f(R)^{c}$.)

Combining this observation with Lemma 3.2 and Lemma 1.7, we have

$$
\begin{aligned}
d\left(f_{*}(x), f_{*}(y)\right) & =\sum_{A \in \mathscr{A}} m\left(A\left(f\left(\gamma_{1}\right), f\left(\gamma_{2}\right)\right)\right) \\
& =\sum_{A \in \mathscr{A}} m\left(f\left(A\left(\gamma_{1}, 2\right)\right)\right) \\
& =\sum_{A \in \mathscr{A}} k \cdot m\left(A\left(\gamma_{1}, \gamma_{2}\right)\right) \\
& =k \cdot d(x, y),
\end{aligned}
$$

where the definition of $A\left(\gamma_{1}, \gamma_{2}\right)$ is similar to that of $A(z, w)$. By the continuity, this also holds for all $x, y \in T^{\prime}$.

Thus the proof of Theorem 3.6 is completed.

\section{Further properties of the tree}

In this section, we start with a dynamical system on a tree $(T, d, F)$ satisfying (a)-(f) of Theorem 3.6, not a priori a tree associated to Herman rings.

Definition 4.1. We assume that $\operatorname{Sing}(T, F)$ denotes the smallest set satisfying (c) and containing end points and branch points. A point of $\operatorname{Sing}(T, F)$ is called a singular point and $I_{i j}$ satisfying (d) periodic arcs. We denote by $\beta(x, Y)$ the branch at $x$ intersecting with a subset (or a point) $Y$, if it exists and is unique. For any $x$ and $b \in \mathscr{B}_{x}$,

$$
\begin{gathered}
F(x, b)=\beta(F(x), F(y)) \quad \text { for } y \in b \text { sufficiently near } x, \\
D F(x, b)=\lim _{b \ni y \rightarrow x} D F(y)
\end{gathered}
$$

are well-defined. We sometimes write them as $F(b), D F(b)$. Note that $F(b)$ does not mean the image of $b$ as a set. Periodic arcs $I_{i j}$ can be oriented so that $f$ respects the orientations. Let $\partial^{-} I_{i j}$ be the beginning of $I_{i j}$ and $\partial^{+} I_{i j}$ the end. Hence $\partial I_{i j}=$ $\left\{\partial^{+} I_{i j}, \partial^{-} I_{i j}\right\}$ and $F\left(\partial^{+} I_{i j}\right)=\partial^{+} I_{i j+1}, F\left(\partial^{-} I_{i j}\right)=\partial^{-} I_{i j+1}$.

Proposition 4.2. $F$ is surjective.

This immediately follows from (d) and (f).

Proposition 4.3. (i) On any periodic orbit, $k_{i}^{ \pm}(x)=\#\left\{j \mid \partial^{ \pm} I_{i j}=x\right\}$ are constant. (ii) $\partial^{+} I_{i j} \neq \partial^{-} I_{i j^{\prime}}$ for $j, j^{\prime} \in Z / p_{i}$. 
Proof. (i) This is obvious from (d). (ii) Suppose $\partial^{+} I_{i j}=\partial^{-} I_{i j+1}$ for some $i, j$, hence for all $j \in Z / p_{i} z$. Then $I_{i j} \cup I_{i j+l} \cup I_{i j+2 l} \cup \cdots$ makes a loop in $T$. This contradicts (a).

Proposition 4.4. $F$ has at least one fixed point. In fact if $F\left(x_{0}\right) \neq x_{0}, F$ has a fixed point in $\beta\left(x_{0}, F\left(x_{0}\right)\right)$.

Proof. Suppose $F\left(x_{0}\right) \neq x_{0}$. Let $r: T \rightarrow T^{\prime}=\beta\left(x_{0}, F\left(x_{0}\right)\right) \cup\left\{x_{0}\right\}$ be a retraction such that $\left.r\right|_{T^{\prime}}=i d$ and $r\left(T-T^{\prime}\right)=\left\{x_{0}\right\}$. By the Lefschetz's fixed point theorem, there exists a fixed point $x_{1}$ of $r \circ F ; r \circ F\left(x_{1}\right)=x_{1}$. Then $x_{1} \in \operatorname{Im} r=T^{\prime}$. Since $F\left(x_{0}\right) \in T^{\prime}$, $r \circ F\left(x_{0}\right)=F\left(x_{0}\right) \neq x_{0}$. Hence $x_{1} \neq x_{0}$ and $x_{1}=r \circ F\left(x_{1}\right) \in \beta\left(x_{0}, F\left(x_{0}\right)\right)$. By the definition of $r, r \circ F\left(x_{1}\right)=F\left(x_{1}\right)$ and $F\left(x_{1}\right)=x_{1}$.

Proposition 4.5. Suppose $b \in \mathscr{B}_{x}$ is periodic; $F^{p}(b)=b(p \geqq 1)$. (Of course $F^{p}(x)=x$.) Then, either $x \in I_{i j}$ for some $i, j$ and $b$ intersects int $I_{i j}$, or $D F^{p}(b)>1$. Here $D F^{n}(b)=$ $D F^{n}(x, b)$ are defined by the chain rule $D F^{n}(x, b)=D F^{n-1}(F(x), F(b)) \cdot D F(x, b)$ or equivalently defined by $D\left(F^{n}\right)$ for $\left(T, F^{n}\right)$ which also satisfy (a)-(f).

The proof is easy. One can consider this as a corollary of the following theorem. This theorem is useful to check that examples in $\$ 5$ satisfy (e).

THEOREM 4.6. Suppose ( $T, d, F)$ satisfies (a)-(d). Then the condition (e) is equivalent to $\left(\mathrm{e}_{1}^{\prime}\right)$ and $\left(\mathrm{e}_{2}^{\prime}\right)$ below:

( $\left.\mathrm{e}_{1}^{\prime}\right)$ There exists $N \geq 0$ such that $T=\bigcup_{n=0}^{N} F^{-n}(S)$, where $S=\bigcup_{i j} I_{i j} \cup$ $\left\{x \mid D F(x, b) \geq 2\right.$ for some $\left.b \in \mathscr{B}_{x}\right\}$;

( $\left.\mathrm{e}_{2}^{\prime}\right)$ Any one-sided neighborhood of a folding point of $F$ intersects $\bigcup_{i, j, n \geq 0} F^{-n}$ (int $\left.I_{i j}\right)$.

(A one-sided neighborhood of $x$ is the intersection of a branch at $x$ and a neighborhood of $x$. A folding point is a point where $F$ is not locally injective.)

Proof. If we assume $\left(\mathrm{e}_{1}^{\prime}\right)$ and $\left(\mathrm{e}_{2}^{\prime}\right)$, then any small arc is homeomorphically expanded by $F^{n N}(n=1,2, \ldots)$ until it intersects with $\bigcup_{I_{i j}}$ or folding points, hence (e) follows.

Suppose $(T, d, F)$ satisfies (e). Immediately $\left(\mathrm{e}_{2}^{\prime}\right)$ follows. In order to prove $\left(\mathrm{e}_{1}^{\prime}\right)$, it suffices (because of the compactness of $T$ ) to prove that:

for any $\alpha:\left[0, t_{0}\right] \rightarrow T$, an isometry onto an arc $\left(t_{0}>0\right)$, there exist $m>0$ and $\tau>0$ such that

$$
F^{m} \circ \alpha[0, \tau] \subset S .
$$

$\left(0^{\circ}\right)$ Let $X=\partial S \cup\left\{x \in\right.$ int $S \mid$ there is a folding point $y$ such that $x=F^{n}(y)(n>0)$ and $F^{i}(y) \notin$ int $\left.S(0 \leq i<n)\right\}$, where int $S, \partial S$ denote the interior and the boundary of $S$ as a subset of $T$. Then $X$ is finite. Take $\delta=$ the minimum of the distance between distinct points of $X$. Of course $\delta>0$.

(1 $\left.{ }^{\circ}\right)$ Define

$$
\begin{gathered}
t_{n}=\sup \left\{t \in\left[0, t_{0}\right] \mid F^{n} \circ \alpha \text { is injective on }[0, t]\right. \text { and } \\
\left.F^{i} \circ \alpha(0, t] \cap \text { int } S=\phi \quad(0 \leq i \leq n)\right\} .
\end{gathered}
$$

Then $t_{n} \geq t_{n+1}$ and $t_{n} \rightarrow 0$ as $n \rightarrow \infty$ by our assumption (e). Suppose $t_{n}=0$ for some $n$, then there exists $m(0 \leq m \leq n)$ such that $F^{m} \circ \alpha(0, t] \cap$ int $S \neq \phi$ for any $t$. Since 
$S$ is a union of a finite number of arcs, it follows that $F^{m} \circ \alpha[0, \tau] \subset S$ for some $\tau>0$. Thus the assertion holds.

Therefore, for the proof of the theorem, we shall derive a contradiction from the assumption that

$$
t_{n}>0 \text { for all } n \geq 0 .
$$

$\left(2^{\circ}\right)$ First notice that $\left.F^{n} \circ \alpha\right|_{\left[0, t_{n}\right]}$ is an isometry. Let $y_{n}=F^{n} \circ \alpha\left(t_{n}\right)$. It is easy to check that if $t_{n}<t_{n-1}$, then

$$
y_{n} \in Y=\partial S \cup\{F(z) \mid z \text { is a folding point }\} .
$$

Since $Y$ is finite, there exist $x$ and $b \in \mathscr{B}_{x}$ such that

$$
M=\left\{n \mid y_{n}=x \quad \text { and } \quad b=\beta\left(x, F^{n} \circ \alpha\left[0, t_{n}\right)\right)\right\}
$$

is infinite.

Fix $i \in M$ large enough so that $t_{i}<\delta$ and that $F^{i} \circ \alpha\left(0, t_{i}\right)$ contains no branch point. If $j \in M$ and $i<j$, then

$$
F^{j} \circ \alpha\left[0, t_{j}\right] \subset F^{i} \circ \alpha\left[0, t_{i}\right],
$$

hence

$$
F^{j} \circ \alpha\left[0, t_{j}\right]=F^{i} \circ \alpha\left[t_{i}-t_{j}, t_{i}\right] .
$$

( $\left.3^{\circ}\right)$ Let $k=\min \left\{n \mid n>i, F^{n} \circ \alpha\left[0, t_{i}\right] \cap\right.$ int $\left.S \neq \phi\right\}$, which exists by (e). There exists $s \in\left(0, t_{i}\right)$ such that $F^{k} \circ \alpha(s) \in$ int $S$ and $F^{k} \circ \alpha$ is locally injective at $s$. Let $J$ be the maximal sub-interval of $\left[0, t_{i}\right]$ containing $s$ such that $F^{k} \circ \alpha(J) \subset S$ and $F^{k} \circ \alpha$ is injective on $J$. If $t \in \partial J$ and $t \neq 0, t_{i}$, then either $F^{k} \circ \alpha$ is not injective at $t$ or $F^{k} \circ \alpha(t) \in \partial S$. It follows from the definition of $X$ that

$$
\partial J \subset\left\{0, t_{i}\right\} \cup\left(F^{k} \circ \alpha\right)^{-1}(X) .
$$

By the definition of $k$ and $J, F^{k} \circ \alpha \mid$, is an isometry, hence $\left|F^{k} \circ \alpha(J)\right|=|J|<t_{i}<\delta$. So $\partial J \not \subset\left(F^{k} \circ \alpha\right)^{-1}(X)$, and either $0 \in \partial J$ or $t_{i} \in \partial J$.

If $0 \in \partial J, t_{k}$ musr be zero. Suppose $t_{i} \in \partial J$. Take $j \in M$ large enough so that $i<j$ and $t_{j}<|J|$. Then

$$
F^{j} \circ \alpha\left[0, t_{j}\right]=F^{i} \circ \alpha\left[t_{i}-t_{j}, t_{i}\right] \subset F^{i} \circ \alpha(J)
$$

hence

$$
F^{j+(k-i)} \circ \alpha\left[0, t_{j}\right] \subset F^{k} \circ \alpha(J) \subset S .
$$

Therefore $t_{j+k-i}=0$. In any case, we have led to a contradiction. Thus the assertion is proved, and this completes the proof of Theorem 4.6.

\section{Examples}

Let us see some examples of trees satisfying the condition (a)-(f) in Theorem 2.8. We restrict our attention to the case where there exists only one cycle of periodic arcs, i.e., $l=1$, so we write $I_{j}=I_{i, j}$. It will be proved in [S2] that there exist rational functions which realize these trees.

Explanation. An arrowed segment with number $j(=0, \infty, \ldots p), \stackrel{j}{\rightarrow}$, denotes a periodic arc $I_{j}$; the number $j$ indicates its cyclic order and the arrow its orientation. Letters $a, b, c, d, \ldots$ denote respective segements and also their lengths for notational 
simplicity; $e$ is specially reserved for the length of the periodic arc. A doubled (resp. tripled) line indicates a part on which $D F=2$ (resp. $D F=3)$. On the rest of the tree, $D F=1$.

A white circle $O$ (resp. a doubled circle (O) denotes the projection by $\pi$ of a critical point (resp. more than two critical points) of a rational function which realizes the tree. $\alpha$ denotes a fixed point.

The map $F$ on the tree is supposed to be the simplest one which sends each periodic arc $j$ to $j+1$, respecting the orientation.

As a matter of course, it is a problem whether or not one can define such an $F$ consistent with the given $D F$ as above. In fact, from the condition (c), we conclude some relations between the lengths of segments. See Examples 3 and 4 . Once the lengths are determined, it is not so difficult to verify the conditions (a)-(f) (using Theorem 4.6 for (e)).

Examples 1 and 2. $T_{1}$ and $T_{2}$ are obtained from the rational functions in Theorems 5(A) and (B) of [S1]. $T_{1}$ is the simplest tree with periodic arcs of period $p . T_{2}$ is isometric to $[-2 e, 2 e]$, and $F(x)=x+3 e$ on $[-2 e,-e] ; F(x)=-2 x$ on $(-e, e)$; $F(x)=x-3 e$ on $[e, 2 e]$.

Example 3. Let us determine the lengths $a, b, c, d$. First, since the branch at $\alpha$ on the right is mapped as in figure 6 , we have

$$
a=b \quad \text { and } \quad d=e+b+a .
$$

Similarly, noting that $D F=2$ on the segments $b$ and $c$, we have

$$
2 b=c \text { and } 2 c=a+e+d .
$$

Solving these equations, we conclude that

$$
a=b=2 e, c=4 e, d=5 e \text { and } e>0 \text { is arbitrary. }
$$

Conversely provided these relations, it is easy to see that $\left(T_{3}, F\right)$ satisfies the condition (a)-(f). Furthermore, it is shown [S2] that $T_{3}$ can be realized by a rational function of degree 3.

Example 4. For $T_{4}$, as in Example 3, we have $a=b$ and $2 b=e+a+e+b+e$, hence $e=0$. Therefore this is impossible. However, we can make it possible by setting $D F=3$ on the segment $b$, as $T_{4}^{\prime}$. Then $a=b=3 e . T_{4}^{\prime}$ is realizable by a function of degree 5 .

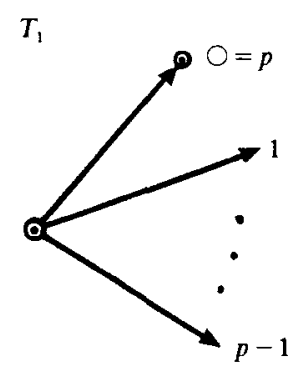

$T_{2}$

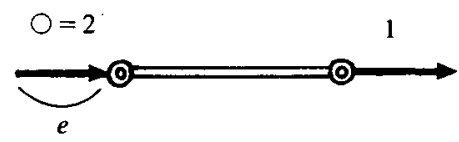

Figure 4 


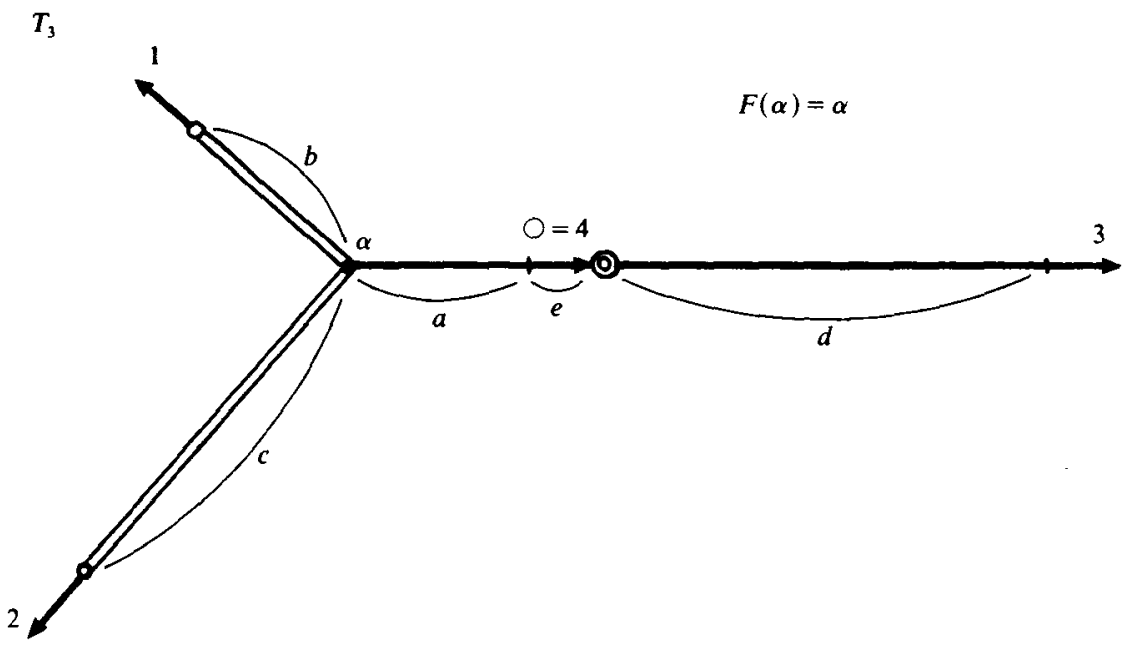

Figure 5

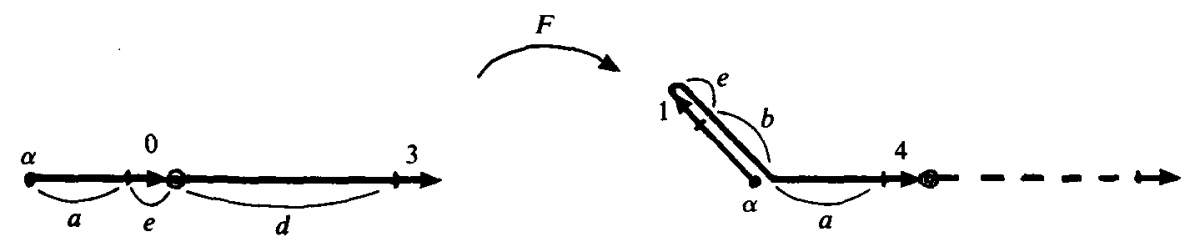

Figure 6
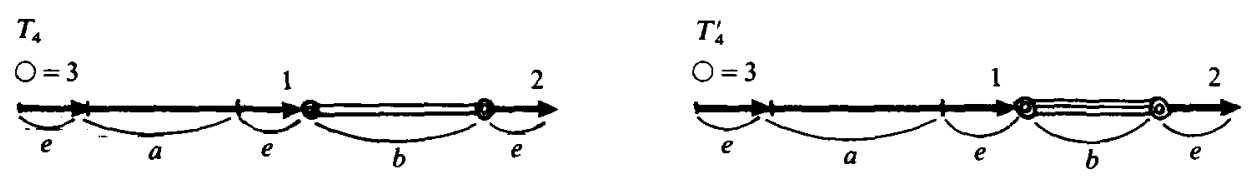

Figure 7

Example 5. With appropriate lengths, $T_{5}$ satisfies (a)-(f). Although it looks complicated, $T_{5}$ is proved to be realizable by a rational function of degree 3 .

\section{Generalization}

We can generalize the construction of $T_{f}$ in $\S 3$, changing $B$ and $\mathscr{A}_{0}$ in Definition 3.1 and also $m(\cdot)$.

Change of $B$. As the set $B$, one can take any non-empty closed set consisting of a finite number of components, provided that $f(B) \subset B$. In this case, Theorem $2.8(f)$ does not necessarily hold. If $B$ has a one point component, then the resulting tree might be of infinite length (see (2.10) Degenerate Case).

Especially, one may add to the original $B$, (super-) attractive basins, parabolic basins, Siegel disks, (or non-repulsive periodic points) and a finite number of their pre-images. 


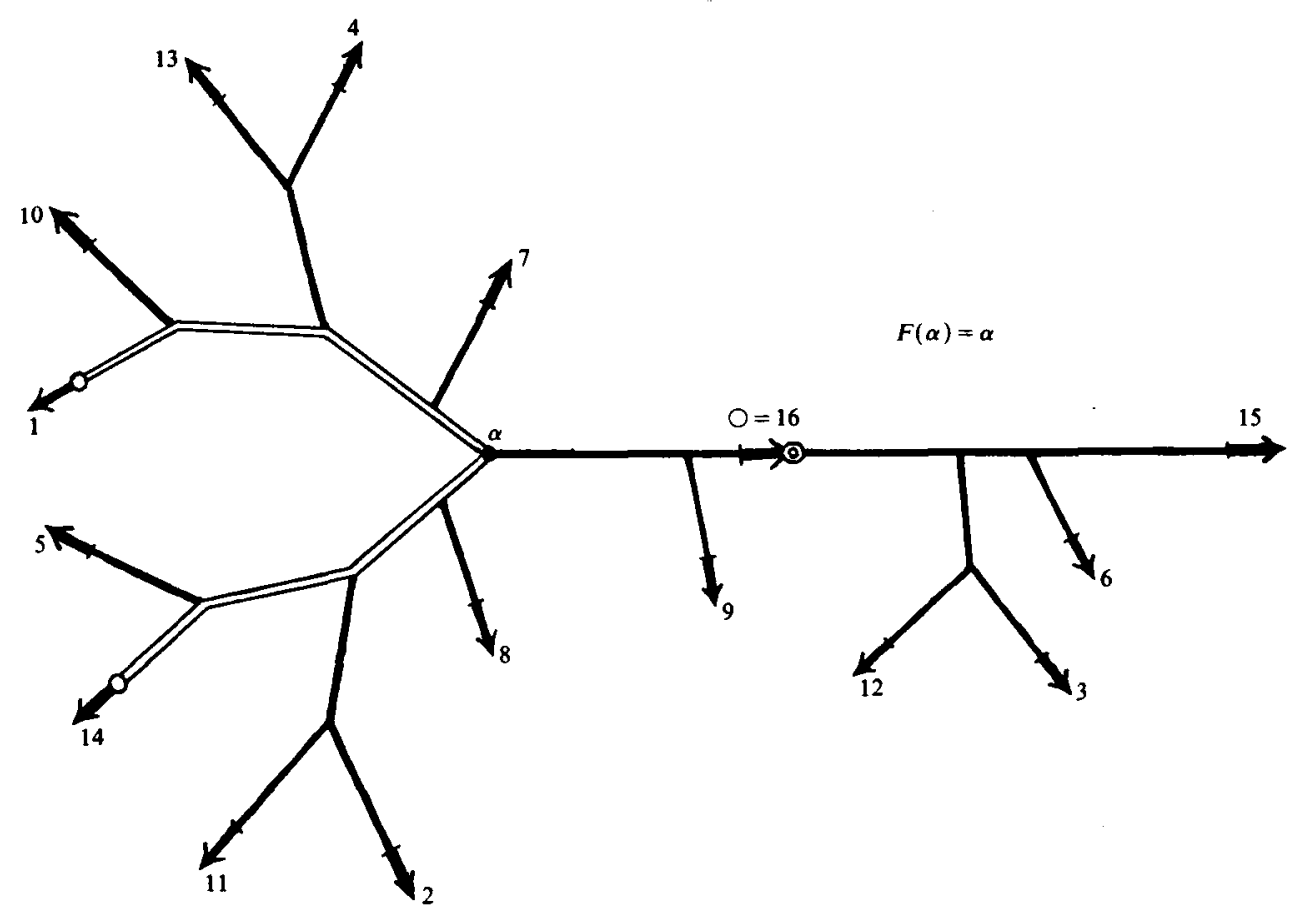

Figure 8

Change of $\mathscr{A}_{0}$. We can define trees related to attractive basins, superattractive basins and Siegel disks.

Let us consider the case of attractive basin. Near an attractive periodic point of period $p, f^{p}$ is conformally conjugate to $z \rightarrow \lambda z$ on $\Delta=\{z \in \mathbb{C}|| z \mid<1\}$ with $0<|\lambda|<1$. Let $C$ be the set of points of $\Delta$ corresponding to the generalized orbits (i.e. forward orbits and their pre-images) of critical points. We may assume $C \cap\{z|| z|=| \lambda \mid\} \neq \phi$. Define

$$
\mathscr{A}_{0}=\left\{\text { components of } \Delta-\left(\{0\} \cup \bigcup_{\zeta \in C}\{z|| z|=| \zeta \mid\}\right)\right\} .
$$

Then it is easy to see that $\mathscr{A}_{0}$ consists of annuli and that if $A \in \mathscr{A}_{0}, f(A) \in \mathscr{A}_{0}$. In this case, we have to add to $B$ the cycle of the attractive periodic points.

In a similar manner, we can define $\mathscr{A}_{0}$ related to superattractive basins or Siegel disks, using conformal conjugacies to $z \rightarrow z^{k}$ or $z-\mathrm{e}^{2 \pi i \theta_{z}}$ on $\{z|| z \mid<1\}$, respectively, where $k$ is an integer $\geq 2$ and $\theta$ an irrational.

If the $\mathscr{A}_{0}$ is changed as above, then Theorem $2.8(\mathrm{~d})$ has to be changed as follows. In the case of (super)attractive basin, let $I_{i j}$ be the projections by $\pi$ of sufficiently small closed neighborhoods of the (super)attractive periodic points such that $f_{*}\left(I_{i j}\right) \subset$ $I_{i j+1}$. In the case of Siegel disk, let $I_{i j}$ be projections by $\pi$ of the closures of Siegel disks such that $f_{*}\left(I_{i j}\right)=I_{i j+1}$. Then each $I_{i j}$ is isometric to the infinite interval $[0,+\infty]$. Moreover, $f_{*}^{p_{i}}$ on $I_{i j}$ is conjugate to

$$
x \rightarrow x+\frac{1}{2 \pi} \log 1 /|\lambda|
$$


in the case of attractive basin, $x \rightarrow k x$ in the case of superattractive basin, and $x \rightarrow x$ in the case of Siegel disk.

It is also possible to utilize a subfamily $\mathscr{A}_{0}^{\prime}$ of $\mathscr{A}_{0}$ provided that $\mathscr{A}_{0}^{\prime}$ is $f$-invariant, i.e. if $A \in \mathscr{A}_{0}^{\prime}, f(A) \in \mathscr{A}_{0}^{\prime}$.

Change of $m(\cdot)$. Instead of the modulus $m(\cdot)$, one can use a weighted modulus, that is, a function $m: \mathscr{A} \rightarrow \mathbb{R}_{+}$provided that if $f: A_{1} \rightarrow A_{2}$ is a covering map of degree $k$ for $A_{1}, A_{2} \in \mathscr{A}$, then $m^{\prime}\left(A_{2}\right)=k m^{\prime}\left(A_{1}\right)$.

Example. Let us see an example of a tree associated with a superattractive basin. Let

$$
f(z)=c\left(\frac{z}{1+z^{2}}\right)^{3},
$$

where $|c| \geq 67$. Then $f$ has a superattractive fixed point $0 ; f(0)=0, f^{\prime}(0)=0$ and $f(\infty)=0$. Critical points of $f$ are $0, \infty$ and \pm 1 . It follows from the condition on $c$ that $f( \pm 1)$ are in the same component of $\bar{C}-J_{f}$ as $\infty$. Consider the tree $T_{f, S A B}$ associated to the superattractive basin with $B=\{0\} \cup\{\infty\}$. It can be proved without effort that

$$
\begin{gathered}
T_{f, S A B}=R \cup\{ \pm \infty\} \text { (isometric); } \\
\pi(0)=-\infty \quad \text { and } \pi(\infty)=+\infty ; \\
f_{*}: x \rightarrow 3 a / 2-3|x-a / 2| \text { on } \mathbb{R}(a>0) \text { and } f_{*}( \pm \infty)=-\infty .
\end{gathered}
$$

Furthermore $\pi\left(J_{f}\right)=a C$, where $C(\subset[0,1])$ is the Cantor's ternary set. It can also be shown that $J_{f}$ itself is homeomorphic to a cartesian product of $a C$ and the unit circle $\{|z|=1\}$ and that $\left.f\right|_{J_{f}}$ is conjugate to $\left(\left.f_{*}\right|_{a C}\right) \times\left(z \rightarrow z^{3}\right)$ on the product.

\section{The proof of the statements in $\$ 2$}

Proof of Lemma 2.2. Let $\mathscr{A}_{i j}(i \neq j)$ be the collection of $A \in \mathscr{A}$ separating $B_{i}$ and $B_{j}$. Since $B_{i}$ and $B_{j}$ are disjoint connected closed sets, there exists a unique component $R$ of $\bar{C}-B_{i} \cup B_{j}$, such that $\bar{R} \cap B_{i} \neq \phi$ and $\bar{R} \cap B_{j} \neq \phi$. Then $R$ is an annulus and every $A \in \mathscr{A}_{i j}$ is an annulus in $R$ separating $\partial R$. By Lemma 1.8,

$$
\sum_{A \in A_{i j}} m(A) \leq m(R)<\infty \text {. }
$$

It follows from the Condition (*) that $\mathscr{A} \subset \bigcup_{i j} \mathscr{A}_{i j}$. Hence

$$
\sum_{A \in \mathscr{A}} m(A) \leq \sum_{i j} \sum_{A \in \mathscr{A}_{i j}} m(A)<\infty
$$

By Lemma 1.6(ii), $m(A(x, y)) \leq m(A)$, therefore $d(x, y)<\infty$.

Proof of Lemma 2.3. It is obvious that $d(x, x)=0$ and $d(x, y)=d(y, x)$. If $w \in A(x, z)$ for $A \in \mathscr{A}$, then $y$ sits either in the component of $A[w]^{c}$ containing $x$, in the other component containing $z$ or on $A[w]$. Accordingly $w \in A(y, z), A(x, y)$ or $A[y]$, where $A[y]=\phi$ if $y \notin A$. Thus

$$
A(x, z) \subset A(x, y) \cup A[y] \cup A(y, z),
$$

and from Lemma 1.6(ii),

$$
m(A(x, z)) \leq m(A(x, y))+m(A(y, z)) \text {. }
$$

Summing them up for $A \in \mathscr{A}$, we obtain the triangle inequality. 
LEMMA 7.1. If $x$ and $z$ are separated by $\gamma \in \Gamma$, then for $y \in \gamma$,

$$
d(x, z)=d(x, y)+d(y, z)
$$

Proof. Suppose that $\gamma$ separates $x$ and $z$ and that $y \in \gamma$. If $A[w](w \in A \in \mathscr{A})$ separates $x$ and $y$ (resp. $y$ and $z$ ), then it must be in the same component of $\gamma^{c}$ as $x$ (resp. $z$ ) and separate $x$ and $z$. Hence

$$
A(x, y) \cap A(y, z)=\phi \text { and } A(x, y) \cup A(y, z) \subset A(x, z)
$$

Then (1) in the proof of Lemma 2.3 becomes an equality and the right-hand side is a disjoint union. Therefore (2) also becomes an equality and the desired equality holds.

Proof of Lemma 2.4. In view of Lemma 2.3, it suffices to prove that:

For any $\varepsilon>0$ and $x \in \overline{\mathbb{C}}$, there exists a neighborhood $U$ of $x$ such that $d(x, y)<\varepsilon$ if $y \in U$.

If $x \in A$ for some $A \in \mathscr{A}$, then take

$$
U=\phi_{A}^{-1}\left(\left\{z\left|\max \left\{r_{A}, e^{-2 \pi} \varepsilon\left|\phi_{A}(x)\right|\right\}<\right| z \mid<\min \left\{1, e^{2 \pi \varepsilon}\left|\phi_{A}(x)\right|\right\}\right\}\right) .
$$

Then the assertion holds, since $A^{\prime}(x, y)=\phi$ for $y \in U$ and $A^{\prime} \in \mathscr{A}, A^{\prime} \neq A$.

If $x \notin \cup \mathscr{A}=\cup_{A \in \mathscr{A}} A$, then one can choose $A_{i} \in \mathscr{A}(i=1, \ldots, n)$ and $A_{i}^{\prime}=$ $\phi_{A_{i}}^{-1}\left(\left\{r_{i}^{\prime}<|z|<r_{i}^{\prime \prime}\right\}\right)$ with $r_{A_{i}}<r_{i}^{\prime}<r_{i}^{\prime \prime}<1$ so that

$$
\sum_{A \in \mathscr{A}-\left\{A_{i}\right\}} m(A)+\sum_{i=1}^{n}\left(m\left(A_{i}\right)-m\left(A_{i}^{\prime}\right)\right)<\varepsilon .
$$

Note that $x \notin \bigcup_{i} \bar{A}_{i}^{\prime}$. Let $U$ be the component of $\overline{\mathbb{C}}-\bigcup_{i} \bar{A}_{i}^{\prime}$ containing $x$. If $y \in U$, then $A_{i}(x, y) \subset A_{i}-A_{i}^{\prime}$ and $m\left(A_{i}(x, y)\right) \leq-m\left(A_{i}^{\prime}\right) m\left(A_{i}^{\prime}\right)$. Therefore

$$
\begin{aligned}
d(x, y) & =\sum_{A \in \mathscr{A}-\left\{A_{i}\right\}} m(A(x, y))+\sum_{i=1}^{n} m\left(A_{i}(x, y)\right) \\
& \leq \sum_{A \in \mathscr{A}-\left\{A_{i}\right\}} m(A)+\sum_{i=1}^{n}\left(m\left(A_{i}\right)-m\left(A_{i}^{\prime}\right)\right)<\varepsilon .
\end{aligned}
$$

In any case, the assertion is proved.

The proof of Lemma 2.6 is left to the reader. (Use Lemmas 2.3 and 2.4.) Before proving Theorem 2.8, we prove Lemma 2.9 .

Proof of Lemma 2.9. If $x \in A$ and $y \notin A[x]$, then for some $\varepsilon>0$,

$$
A(x, y) \subset \phi_{A}{ }^{-}\left(\left\{\zeta|r<| \zeta \mid<r e^{\varepsilon}\right\}\right) \text { or } \phi_{A}^{-}\left(\left\{\zeta\left|r e^{-\varepsilon}<\right| \zeta \mid<r\right\}\right),
$$

where $r=\left|\phi_{A}(x)\right|$. Hence $d(x, y) \geq m(A(x, y))>0$. It immediately follows that $\pi^{-1}(\pi(x))=A[x]$ and $\pi^{-1}(\pi(A))=A$.

If $x, y \in A$ and $A^{\prime} \neq A$, then $A^{\prime}(x, y)=\phi$. Hence by Lemma 1.7(ii),

$$
d(x, y)=m(A(x, y))=\frac{1}{2 \pi}|\log | \phi_{A}(x)|-\log | \phi_{A}(y)|| .
$$

Thus $x \rightarrow\left(1 / 2 \pi \log \left|\phi_{A}(x)\right|\right.$ gives an isometry to an interval.

Proof of Theorem 2.8. $(\gamma)$ It is easy to see that for any $z \in \overline{\mathbb{C}}$ there exists $z^{\prime} \in \overline{\cup \mathscr{A}}$ such that $d\left(z, z^{\prime}\right)=0$. Hence $T(\mathscr{A})=\pi(\overline{\mathbb{C}})=\pi(\bigcup \mathscr{A})$. By Lemma $2.4, \pi$ is continuous, so $\pi(\bar{\cup})=\overline{\pi(\bigcup \mathscr{A})}$. Then we have $\overline{\pi(\bigcup \mathscr{A})}=T(\mathscr{A})$. 
Proof of $(\alpha)$. For a simple closed curve $\gamma$ and a set $X \subset \overline{\mathbb{C}}$, let $\mathscr{C}(\gamma, X)$ denote the component of $\gamma^{c}$ containing $X$, if it exists. We use a similar notation $\mathscr{C}(A, X)$ for an annulus $A$. If $\gamma_{1}$ and $\gamma_{2}$ are disjoint simple closed curves, let $R\left(\gamma_{1}, \gamma_{2}\right)$ denote the annulus bounded by $\gamma_{1}$ and $\gamma_{2}$.

If $X$ and $Y$ are unions of some $B_{i}$ such that $X \neq \phi, Y \pm \phi, X \cap Y=\phi$ and $X \cup Y=B$, then $\{X, Y\}$ is called a partition. For a partition $\{X, Y\}$, set

$$
\begin{gathered}
\mathscr{A}(X, Y)=\{A \in \mathscr{A} \mid A \text { separates } X \text { and } Y\}, \\
\Gamma(X, Y)=\{A[z] \mid A \in \mathscr{A}(X, Y), z \in A\} \subset \Gamma .
\end{gathered}
$$

Let us consider a partition $\{X, Y\}$ for which $\mathscr{A}(X, Y) \neq \phi$. Define

$$
C^{+}=\bigcap_{A \in \mathscr{A}(X, Y)} \mathscr{C}(A, X), \quad C^{-}=\bigcap_{A \in \mathscr{A}(X, Y)} \mathscr{C}(A, Y), \quad E=\overline{\mathbb{C}}-\left(C^{+} \cup C^{-}\right) .
$$

LemMA 7.2. (i) $C^{+}, C^{-}$are connected closed set containing $X, Y$, respectively. $E$ is an annulus.

(ii) If $A \notin \mathscr{A}(X, Y)$, then $A \subset C^{+}$or $A \subset C^{-}$.

(iii) If $\gamma \in \Gamma$ separates $x, y \in E$, then $\gamma \in \Gamma(X, Y)$.

Proof. (i) There exist $\gamma_{n}^{\prime}, \gamma_{n}^{\prime \prime} \in \Gamma(X, Y)(n=1,2, \ldots)$ such that

$$
C^{+}=\bigcap_{n} \frac{\left\{\mathscr{C}\left(\gamma_{n}^{\prime}, X\right)\right\},\left\{\mathscr{C}\left(\gamma_{n}^{\prime \prime}, Y\right)\right\} \quad \text { are decreasing and }}{\mathscr{C}\left(\gamma_{n}^{\prime}, X\right)}, \quad C^{-}=\bigcap_{n} \overline{\mathscr{C}\left(\gamma_{n}^{\prime \prime}, Y\right)}, \quad E=\bigcup_{n} R\left(\gamma_{n}^{\prime}, \gamma_{n}^{\prime \prime}\right) .
$$

As each $C^{+}$is an intersection of decreasing connected compact sets, it is connected. Similarly, $E$ is connected. (ii) and (iii) Suppose $A \in \mathscr{A}-\mathscr{A}(X, Y)$. Then $A$ must separate $X$ or $Y$. We may assume that $A$ separates $X$. Then $A \subset \mathscr{C}(\gamma, X)$ for $\gamma \in \Gamma(X, Y)$. Thus $A \subset C^{+}$. Since $\bar{E}$ is connected and $\bar{E} \subset\left(C^{+}\right)^{c} \subset A^{c}, \gamma=A[z]$ $(z \in A)$ cannot separate any two points of $\bar{E}$.

LEMMA 7.3. Fix $z_{0} \in X$ and denote $\zeta=\pi\left(z_{0}\right)$. Then, for any $x, y \in \bar{E}$.

$$
d(x, y)=\left|d\left(z_{0}, x\right)-d\left(z_{0}, y\right)\right| \text {. }
$$

Hence

$$
e=\pi(\bar{E}) \ni \xi \rightarrow \bar{d}(\zeta, \xi) \in \mathbb{R}
$$

gives an isometry onto a closed interval. Furthermore, its interior and end points correspond to int $e=\pi(E)$ and $\partial^{ \pm} e=\pi\left(\partial E \cap C^{ \pm}\right)$.

Proof. Suppose $x, y \in \bigcup \mathscr{A}(X, Y)$ and $x \in \gamma_{1}, y \in \gamma_{2}, \gamma_{1} \neq \gamma_{2}$. By Lemma 7.2(iii), $\gamma_{1}$, $\gamma_{2} \in \Gamma(X, Y)$, so $R\left(\gamma_{1}, \gamma_{2}\right) \cap B$ must be empty (otherwise $\gamma_{1}$ and $\gamma_{2}$ give rise to different partitions of $B$ ). Therefore, either $\gamma_{1}$ separates $z_{0}$ and $y$, or $\gamma_{2}$ separates $z_{0}$ and $x$. Then (4) follows from Lemma 7.1. It holds on $\overline{\mathscr{A}(X, Y)}$ by continuity, and also on $\bar{E}$ since for any $x \in \bar{E}$ there exists $x^{\prime} \in \overline{\bigcup A(X, Y)}$ such that $d\left(x, x^{\prime}\right)=0$. It can be checked, using (3) that int $e=\pi(E), \partial^{ \pm} e=\pi\left(\partial E \cap C^{ \pm}\right)$.

Let $\left\{X_{k}, Y_{k}\right\}(k=1, \ldots, K)$ be all the partition for which $\mathscr{A}_{k}=\mathscr{A}\left(X_{k}, Y_{k} \neq \phi\right.$. (If necessary, normalize by $B_{1} \subset X_{k}$.) Of course there are only finitely many partitions, because of the finiteness of $\left\{B_{i}\right\}$. Denote the sets defined as above for $\left\{X_{k}, Y_{k}\right\}$ by $C_{k}^{ \pm}, E_{k}, e_{k}=\pi\left(\bar{E}_{k}\right), \partial^{ \pm} e_{k}$. 
LEMMA 7.4. $E_{k} \cap E_{l}=\phi$ and int $e_{k} \cap$ int $e_{l}=\phi$ whenever $k \neq l$.

Proof. Suppose $k \neq l$ and $\gamma, \gamma^{\prime} \in \Gamma\left(X_{k}, Y_{k}\right)$. By the proof of Lemma 7.2(ii), for $s=+$ or - , both $\gamma$ and $\gamma^{\prime}$ are contained in $C_{l}^{s}$. We may assume $s=+$. Then $\left(E_{l} \cup C_{l}^{-}\right) \cap$ $\left(\gamma \cup \gamma^{\prime}\right)=\phi$. Recall that $E_{l} \cup C_{l}^{-}$is connected and contains $Y_{l}(\subset B)$. Hence $E_{l} \cup C_{l}^{-}$ does not intersect with $R\left(\gamma, \gamma^{\prime}\right)$. Therefore, by (3), $E_{k} \cap E_{l}=\phi$.

It follows from (3), Lemma 2.9 and 7.1 that $\pi\left(E_{k}\right) \cap \pi\left(E_{l}\right)=\phi$.

Let $F$ be the collection of the non-empty intersections of the form $\cap{ }_{k} C_{k}^{s_{k}}\left(s_{k}=+\right.$ or -$)$.

LemMa 7.5. Suppose $F \in \mathscr{F}$. Then $F$ is connected; $A \cap F=\phi$ for all $A \in \mathscr{A} ; \pi(F)$ is a point; $\pi^{-1}(\pi(F))=F$; there is $k$ such that $\partial E_{k} \cap F \neq \phi$, hence $\pi(F)=\partial^{+} e_{k}$ or $\partial^{-} e_{k}$.

As in Lemma 7.2(i), $F$ is proved to be connected. The proof of the rest is easy.

It follows that $\mathscr{F}$ consists of components of $\overline{\mathbb{C}}-\bigcup_{k} E_{k}$. Combining all results, it is proved that $T(\mathscr{A})$ is the union of the arcs $e_{k}(k=1, \ldots, K)$ with some of end points $\partial^{ \pm} e_{k}$ identified. So this is nothing but a one-dimensional simplicial complex. It is connected, since $\pi: \overline{\mathbb{C}} \rightarrow T(\mathscr{A})$ is continuous and $\overline{\mathbb{C}}$ is connected.

Suppose $\xi \in \pi(\cup \mathscr{A})$. Then $\pi^{-1}(\xi)(\in \Gamma)$ divide $\overline{\mathbb{C}}$ into two components $D, D^{\prime}$. By Lemmas 2.9 and 7.1, $\pi(D) \cap \pi\left(D^{\prime}\right)=\phi$. Hence $T(\mathscr{A})-\{\xi\}=\pi(D) \cup \pi\left(D^{\prime}\right)$ is disconnected. Since $\pi(\cup \mathscr{A})$ is dense, $T(\mathscr{A})$ cannot contain any loop.

Thus we conclude that $T(\mathscr{A})$ is a tree, and $(\alpha)$ is proved.

Proof of $(\beta)$. Let $L$ be an arc in $T(\mathscr{A})$ with end points $\xi, \zeta$. If $\eta \in$ int $L \cap \pi(\cup \mathscr{A})$, $\xi$ and $\zeta$ belong to different components of $T(\mathscr{A})-\{\eta\}$. So $\pi^{-1}(\xi)$ and $\pi^{-1}(\zeta)$ must belong to different components of $\overline{\mathbb{C}}-\pi^{-1}(\eta)$ (see the last part of the proof of $(\alpha)$ ), that is to say, $\pi^{-1}(\eta)$ separates $\pi^{-1}(\xi)$ and $\pi^{-1}(\zeta)$. By Lemma 7.1, we have $d(\xi, \zeta)=d(\xi, \eta)+d(\eta, \zeta)$. It follows, as in the proof of Lemma 7.3, that this equality holds for all $\eta \in L$. Therefore $d$ is a linear metric.

Proof of $(\delta)$. Suppose $\xi$ is an end point of $T(\mathscr{A})$. It is easily proved that $\pi^{-1}(\xi)=C_{k}^{s}$ for some $k$ and $s=+$ or - . The $C_{k}^{s}$ contains some $B_{i}$, hence $\xi=\pi\left(B_{i}\right)$.

Thus Theorem 2.8 is proved.

\section{REFERENCES}

[A1] L. V. Ahlfors. Complex Analysis. McGraw-Hill: New York, 1953.

[A2] L. V. Ahlfors. Lectures on Quasiconformal Mappings. Van Nostrand: New York, 1966.

[B] P. Blanchard. Complex analytic dynamics on the Riemann sphere. Bull. AMS. 11 (1984), 85-141.

[D] A. Douady. Disques de Siegel et anneaux de Herman. Séminaire N. Bourbaki, exposé 677 (1986/1987).

[DH] A. Douady et al. Etudes dynamiques des polynômes complexes I, II. Publ. Math. d'Orsay 84-02 (1984), 85-05 (1985).

[H] M. R. Herman. Exemples de fractions rationnelles ayant une orbite dense sur la sphère de Riemann. Bull. Soc. Math. France 112 (1984), 93-142.

[S1] M. Shishikura. On the quasiconformal surgery of the rational functions. Ann. Scient. Ec. Norm. Sup. (4) t.20 (1987), 1-29.

[S2] M. Shishikura. Construction of Herman rings from trees. In preparation. 